\title{
La logística inversa en el sector manufacturero y su impacto en el medio ambiente
}

\author{
Inverse logistics in the manufacturing sector and its impact on the environment
}

${ }^{1}$ Elard Thamar Salas Valdivia
Universidad Privada de Tacna. Tacna - Perú.
ORCID: 0000-0001-6709-3836
E-mail: elardsalasv@hotmail.com

\section{RESUMEN}

El objetivo de la presente investigación fue resaltar la importancia de la gestión de Logística Inversa como un mecanismo de aprovechamiento de recursos desechables para darles oportunidad de valor económico. La investigación se basó en la existencia de un incremento en el beneficio de Logística Inversa en las relaciones entre empresa y medio ambiente. Las tendencias ambientales, culturales y sociales, y la escasez de los recursos naturales exigen a las empresas de Industria Gráfica optimizar a través de la Logística Inversa, como apoyo no solo en su desempeño operativo, sino más aún, el grado de competitividad razonable en sus recursos y capacidades. La metodología utilizada consistió en la revisión documental en relación al tema de Logística Inversa que existe en la literatura, en el periodo comprendido entre 1990 y 2017 . Como resultado se pudo resaltar que, la estrategia de la organización debería centrarse en las competitividades principales que esta posee, y que podría maximizar el valor de la organización. Como conclusión se pudo enfatizar las ventajas económicas que son ampliamente considerables para las empresas, y sus implicancias en el tema de actividades económicas ambientalmente sostenibles.

Palabras clave: Impacto ambiental, logística inversa, teoría basada en recursos y capacidades.

\begin{abstract}
The aim of this research was to highlight the importance of Reverse Logistics management as a mechanism to take advantage of disposable resources to give them an opportunity of economic value. The research was based on the existence of an increasing in the benefit of Reverse Logistics in the relationship between the company and the environment. Environmental, cultural and social trends, and the scarcity of natural resources require Graphics Industry companies to optimize through Reverse Logistics, as a support not only in their operational performance, but even more, the degree of reasonable competitiveness in their resources and capabilities. The methodology used consisted of the documentary review in relation to the Reverse Logistics issue that exists in the literature, in the period between 1990 and 2017. As a result, it could be highlighted that the organization's strategy should focus on the main competitiveness it has and that it could maximize the value of the organization. In conclusion, it was possible to emphasize the economic advantages that are widely considerable for companies and their implications on the issue of environmentally sustainable economic activities.
\end{abstract}

Keywords: Environmental impact, reverse logistics, theory based on resources and capacities

\section{INTRODUCCIÓN}

Desde la presentación de la Teoría de los Recursos y Capacidades las empresas investigan ventajas competitivas sostenibles en sus recursos y capacidades, analizándolos y potenciándolos (Amit \& Schoemaker, 1993).

Por ello, la estrategia de la organización debe centrarse en las competencias fundamentales que ésta posee y que permite maximizar el valor de la organización (Prahalad y Hamel, 1990).

Existe un interés, cada vez mayor, en la interacción entre empresa y medio ambiente, y se aprecia una percepción crítica por medio de los agentes sociales. En materia de análisis cuando determinamos cuál es el papel que debe 
desempeñar la empresa en su relación con el entorno ambiental, especialmente porque existe la preocupación de generar bienestar actual y perspectiva de la sociedad en el futuro, es importante la gestión de los residuos generados por las empresas en el ejercicio de su actividad. A lo largo de su proceso de operaciones (aprovisionamiento, producción, almacenaje, distribución), generan multiplicidad de tareas en subproductos, en su primer momento, no teniendo otro destino final que el vertedero.

El propósito es saber de qué manera la aplicación de la Logística Inversa, causada por la recuperación de estos Productos Fuera de Uso (PFU), serán creadores de ventajas competitivas sostenibles para la empresa y para la sociedad.

Igualmente, la consideración de un flujo inverso en la función logística amplificaría las capacidades competitivas de la empresa, en el sentido de incrementar los recursos y capacidades, sobre los que se podrían desarrollar las potencialidades de la organización y conseguir, de esta forma, la ansiada ventaja competitiva sostenible; ya que para satisfacer las necesidades cada vez más exigentes de los clientes, el producto debe ser no sólo de calidad, sino altamente competitivo, estar disponible en el momento y lugar oportuno, y ser respetuoso con el medioambiente. Todo ello implica hacer frente a un incremento de los costes, adaptarse a la normativa creciente en materia medio ambiental y ofrecer todo tipo de políticas de devoluciones.

Las actividades de Logística Inversa requieren de una adecuada Gestión del Conocimiento en todas las fases del retorno del producto que permita resolver los problemas a los que debe hacer frente en todos estos procesos (Wadhwa y Madaan, 2007), y también aproximar los objetivos actuales de satisfacción del cliente y beneficio empresarial a la legislación a favor del medio ambiente.

En este sentido, será fundamental para la organización poder contar con la capacidad de generar nuevo conocimiento que reduzca la elevada incertidumbre de las actividades de Logística Inversa (Drucker, 1993), dotándola de una mayor flexibilidad que amplíe su capacidad de respuesta ante los continuos cambios que en estas actividades se producen. A pesar de la creciente importancia de ambas variables, apenas existen trabajos en la literatura científica que traten de analizar la relación existente entre las mismas, y las posibles implicaciones que de ella puedan surgir. Por este motivo, en este trabajo de investigación nos planteamos analizar la relación entre la creación del conocimiento y la Logística Inversa, y examinar su influencia sobre la flexibilidad de la información y los resultados de la organización.

A inicios de los años 80 la historia de la Logística Inversa se inicia a consecuencia de una serie de protestas protagonizadas por grupos ecologistas que sacudió a los países industrializados, por los daños producidos al medio ambiente, sindicando como responsables de esta situación a las grandes cadenas de distribución, por los daños al medio ambiente, los cuales eran integrados con una gran variedad de atractivos y modernos envases.

Tales movimientos ecologistas y las fuerzas de la oferta y la demanda en las grandes y pequeñas economías, han inducido con fuerza inusitada el mercado del papel reciclado lo que hoy en día existe una serie de productos variados que van desde el papel de oficina hasta bolsas para empacar comidas rápidas, que se elaboran con base en la pulpa de papel reciclado.

Esta situación dada por la conservación del medio ambiente, enfoca dos campos fundamentales, primero evitar la tala de árboles y segundo, la disminución en cantidad y tamaño de rellenos sanitarios o vertederos de basuras como se les conoce comúnmente.

La Logística Inversa surge como una necesidad de reutilización de materiales colocados en nuestro entorno por algún proceso productivo.

La conservación ambiental y reducción del calentamiento global cobra fuerza, especialmente, como tema de preocupación en los países desarrollados, responsables de la mayor producción mundial, así como en países en vías de desarrollo y/o países emergentes que buscan una mejor calidad de vida.

Existe un alto grado de responsabilidad en las empresas productoras, de gran y mediana escala, para la reutilización de aquellos productos que fueron puestos en el mercado tales como las mermas o fallas de producción, incorporándolas como materias primas de sus procesos productivos. Asimismo, estas empresas se encuentran ante una oportunidad de adquisición y mejora en la productividad, a un menor costo que recala en la eficiencia de la empresa y en la imagen institucional que proyecta a la sociedad. 
Ante esto se considera que debemos tomar en cuenta y no dejar de lado antes de hablar de Logística Inversa, la situación actual del mercado, y es que existe un crecimiento de las exportaciones, alternativa de algunos sectores, en especial de mercados emergentes, caso Perú, Chile y Colombia.

Lo que pretenden las organizaciones hoy en día es fidelizar a sus clientes, que cada vez se ponen más agresivas en sus condiciones de venta relativas a devoluciones y reclamaciones.

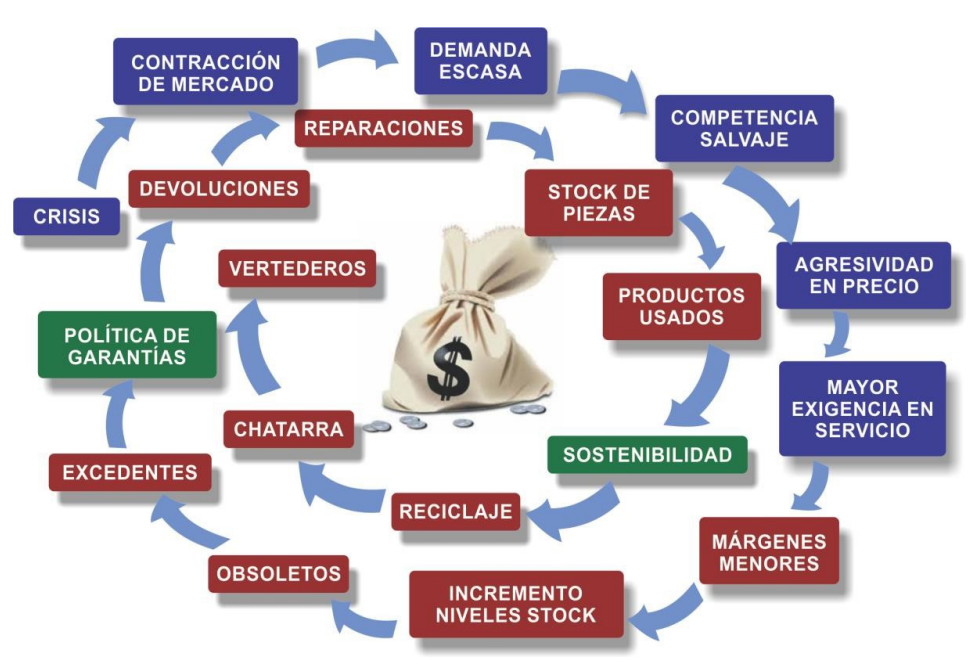

Figura 1. Enfoque de la Logística Inversa - Situación Actual del Mercado

Fuente: II Jornada Logística Inversa - Feb-2013 UNO/Instituto Logístico Tajamar - Madrid, Costos asociados a la Gestión de Stocks y al servicio postventa crecientes.

La Logística Inversa como una segunda oportunidad de negocio, establece ofrecer un cambio constante, resulta que la disponibilidad de recursos no-renovables (metales, petróleo) para los fabricantes de productos originales es crucial para continuar con la creación de valor y rentabilidad. Las tendencias en el mundo globalizado generan la necesidad de nuevas técnicas para atender las políticas y regulaciones sobre el cuidado al medio ambiente.

Políticas más partidarias para la devolución de productos, las regulaciones vigentes y futuras sobre el medio ambiente fuerzan a las empresas a recuperar los productos al final de su ciclo de vida, considerando recuperar algún valor de éstos a través de la remanufacturación, reciclaje, reutilización. El despliegue de protección al ambiente toma su mayor efecto sobre la imagen empresarial y sus productos.

Rogers y Tibben - Lembke (1999) consideran la logística inversa como el proceso de planear, implementar y controlar eficientemente, y a un costo apropiado, los flujos de materias primas, inventario en proceso, bienes terminados e información relacionada desde el punto de origen con el propósito de recuperar el valor primario o disponer adecuadamente de ellos.

Gaytán (2012) constituye que la logística inversa es el proceso de planeación, implementación y control del flujo de materias primas, inventario en proceso y bienes terminados, desde un punto de uso, manufactura o distribución a un punto de recuperación o disposición adecuada. Carter \& Eltram (2006) establece una jerarquía de actividades para el área de logística inversa: Desecho apropiado de residuos, desecho con recuperación de energía, reciclaje, reutilización, reducción en el uso de recursos a través del rediseño del producto.

Problema: El uso de recursos productivos enfrenta hoy en día un problema integral, esto se deriva del análisis interno en tres factores: Vencimiento del producto, orientación a los productos de devoluciones por imperfectos y al impacto ambiental que estos puedan causar. Se ha podido establecer que dentro de sus principales problemas de gestión es la implementación de una adecuada política de un sistema de Logística Inversa, la cual debe considerarse como un objetivo prioritario dentro de la empresa como un factor de negocio muy importante. 
La Logística Inversa ayudará a preservar el medio ambiente mediante, el reciclaje de materiales que ponen en peligro el ecosistema terrestre. A producir una mentalización y colaboración total entre todos los agentes: proveedor, capital Humano, distribución, transporte, y usuario final.

Estos factores impiden el desarrollo de un plan integral a base del conocimiento, de recoger, desmontar y procesar productos usados, parte de productos o materiales con vistas de maximizar el aprovechamiento de su valor y uso sostenible de sus materiales desde el punto de vista de consumo al punto de origen como también asegurar una correcta eliminación de los materiales improductivos.

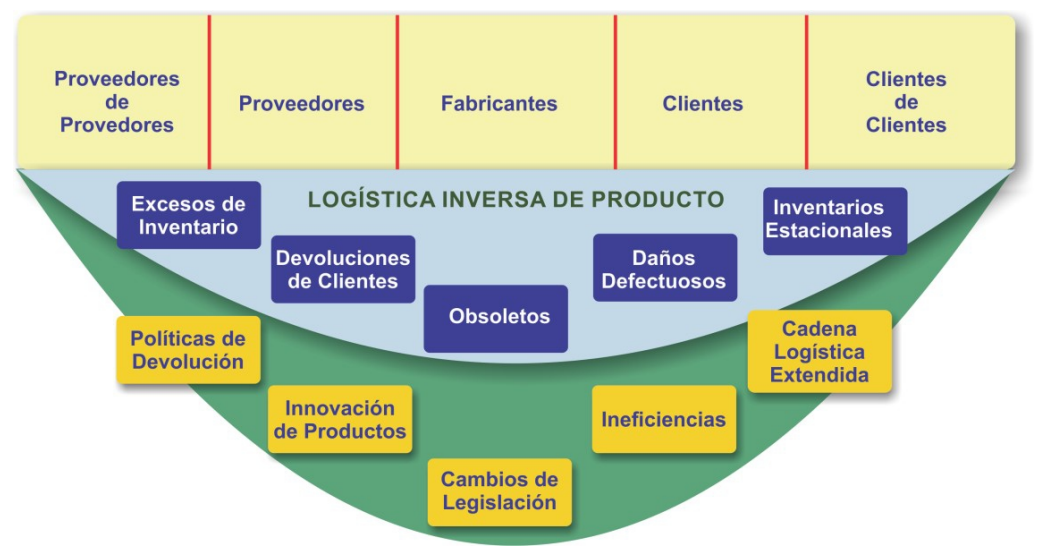

Figura 2. Logística inversa de producto

Fuente: Elaboración propia

La Logística Inversa se encarga de la recuperación y reciclaje de envases, embalajes y residuos peligrosos e incluso se adelanta al fin de vida del producto, con objeto de darle salida en mercados con mayor rotación.

La Logística Inversa gestiona: el retorno de las mercancías en la cadena de suministro, de la forma más efectiva y económica posible, lo que implica en los próximos años una importante modificación de muchos procesos productivos y, además, una oportunidad como nuevo mercado para muchos operadores de transporte, almacenaje y distribución.

La logística inversa supone una importante revolución en el mundo empresarial y, muy probablemente, convertirse en uno de los negocios con mayor crecimiento en el inicio del tercer milenio.

Es una actividad con un enorme potencial de crecimiento definida por expertos como la última frontera para la reducción de costos en las empresas, como también en una importante y novedosa fuente de oportunidades. 
Tabla 1. Definición de Logística Inversa

\begin{tabular}{ll}
\hline Autor & \multicolumn{1}{c}{ Definición } \\
\hline Kroon y Vrijens (1995) & $\begin{array}{l}\text { Gestión Logística y las actividades implicadas en la reducción, gestión y } \\
\text { disposición de residuos y no residuos desechados. Implica que los bienes y } \\
\text { servicios fluyan en sentido contrario a las actividades. }\end{array}$ \\
Fleischmann et al.(1997) & $\begin{array}{l}\text { Proceso por el que mediante la actividad Logística los productos usados de escasa } \\
\text { longevidad pasan a productos utilizados de nuevo en un mercado. }\end{array}$ \\
Carter \& Ellram (2006) & $\begin{array}{l}\text { Logística inversa es el proceso a través del cual las empresas pueden llegar a ser } \\
\text { medioambientalmente más eficientes mediante el reciclaje, reutilización y } \\
\text { reducción de la cantidad de los materiales utilizados. }\end{array}$
\end{tabular}

Krikke (1998)

Recolección, transporte, almacenaje y procesamiento de productos desechados.

Ross (1998)

Supone el traslado de bienes desde su destino típico final a otro, capturando el valor del producto que normalmente se perdería por este traslado.

Rogers y Tibben-Lembke Proyectar y controlar un flujo de materia prima, inventario en proceso, productos terminados e información relacionada desde el punto de consumo hasta el punto de origen de una forma eficiente y lo más económica posible con el propósito de recuperar su valor o el de la propia devolución.

Dowlatshahi (2000)

Proceso en el que el fabricante acepta sistemáticamente productos o partes de productos, readaptados previamente desde el punto de consumo para su reciclaje, refabricación o nueva disposición.

Guide et al. (2000)

La tarea de recuperar productos desechados, incluyendo el embalaje y envío a una central para su reciclaje o reconstrucción.

Tibben-Lembke (2002) La logística inversa gestiona el retorno de las mercancías en la cadena de suministros, de la forma más efectiva y económica posible.

Council of Logistics Rol de la Logística en el reciclaje, disposición de desperdicios y el manejo de Management (2003) materiales peligrosos; una perspectiva más amplia incluye todo lo relacionado con las actividades logísticas llevadas a cabo en la reducción de entrada, reciclaje, substitución y reutilización de materiales y su disposición final.

Stock (2004) La Logística Inversa es un término utilizado frecuentemente para referirse al papel de la Logística en la devolución de productos, reducción de suministros, reciclaje, sustitución y reutilización de materiales, eliminación de desperdicios, reprocesamiento, reparación y refabricación.

A continuación, se presenta la diferencia conceptual entre la logística inversa y la logística directa. 
Tabla 2. Diferencia entre Logística Directa e Inversa. Aspectos esenciales.

\begin{tabular}{|c|c|}
\hline Logística Directa & Logística Inversa \\
\hline Estimación de demanda relativamente cierta & Estimación de demanda más compleja \\
\hline Transporte de uno a muchos, generalmente & Transporte de muchos a uno, generalmente \\
\hline Calidad del producto uniforme & Calidad del producto no uniforme \\
\hline Envase uniforme del producto & Envase a menudo dañado o inexistente \\
\hline Precio relativamente uniforme & Precio dependerá en función de muchos factores \\
\hline Reconocida importancia a la rapidez de entrega & Poca importancia en general de la rapidez de entrega \\
\hline $\begin{array}{l}\text { Costos definidos y monitorizados por sistemas de } \\
\text { contabilidad }\end{array}$ & $\begin{array}{l}\text { Costos inversos son menos visibles y rara vez se } \\
\text { contabilizan }\end{array}$ \\
\hline Gestión de inventarios relativamente sencilla & Gestión de inventario más compleja \\
\hline Ciclo de vida del producto gestionable & Ciclo de vida del producto más complejo \\
\hline Métodos de Marketing bien conocidos & Marketing puede ser complejo por varios factores \\
\hline
\end{tabular}

Las diferencias entre los dos campos, la logística inversa en relación con la logística directa, no necesariamente resulta ser "un cuadro simétrico de distribución directa" (Fleischmann et al., 1997) quien analiza el impacto de los flujos bidireccionales en diseños de red y operacionales. A continuación, se presenta un cuadro comparativo por Tibben-Lembke (2002)

En los modelos tradicionales de inventario, la incertidumbre se enfoca en función de la cantidad de productos demandados y se asume que el precio a que el producto se venderá es conocido. En la logística inversa, la llegada de producto y al precio que se venderá tiende a ser muy el azar, desconocido.

Tabla 3. Costos Logística Inversa vs. Logística Directa.

\begin{tabular}{lc}
\hline Costos de Logística Inversa & Comparación con la logística directa \\
\hline $\begin{array}{l}\text { Transportación } \\
\text { Costo de mantener inventario }\end{array}$ & $\begin{array}{c}\text { Mucho mayor } \\
\text { Merma o (Robo) }\end{array}$ \\
Obsolescencia & Menor \\
Clasificación y diagnóstico de calidad & Puede ser mayor \\
Manipulación & Mucho mayor \\
Reparación y re-empaque & Mucho mayor \\
Cambio de valor en los libros & Significativo para LI, no existente LD \\
\hline
\end{tabular}

Fuente: Adaptado de Tibben Lembke, 2002

En la logística inversa participan actores con diferentes funciones, responsabilidades y niveles estratégicos que permiten lograr sus objetivos, alcanzar los beneficios potenciales y ejecutar los diversos procesos involucrados al mínimo costo y con niveles adecuados de desempeño. Dichos actores pueden ser clasificados como:

a. Actores principales: se consideran los proveedores, distribuidores, minoristas, cliente y la(s) empresa(s) responsable(s) de la recuperación del producto o productor.

b. Actores especializados: prestadores de servicio de transporte, almacenamiento, recicladores, operadores de reprocesamiento o eliminación de desechos, quienes vienen a ser los que ejecutan los procesos específicos de la logística inversa.

c. Actores relacionados: organizaciones gubernamentales, ONG ambientalistas, entre otras, que afectan a la logística inversa de la cadena de suministro, etc.

La Logística Inversa se encarga de la recuperación y reciclaje de envases, embalajes y residuos peligrosos; así 
como de los procesos de retorno de excesos de inventario, devoluciones de clientes, productos obsoletos e inventarios estacionales. Incluso se adelanta al fin de vida del producto, con objeto de darle salida en mercados con mayor rotación.

Llevar a cabo una buena Logística Inversa conlleva una importante tarea de planificación para llevar a cabo la retirada del producto de los puntos de venta. Incluirla dentro del resto de la cadena de suministro, provoca sinergias con la logística directa y llega a acuerdos de nivel de servicio entre el operador y el cliente. La magnitud y el impacto de la Logística Inversa difieren en función del tipo de industria y de los modos de distribución elegidos, pero lo que está despejado es que la Logística Inversa tiene un importante peso en la economía y este peso está aumentando en los últimos años.

Hay que tener en cuenta, que, para algunas empresas, la logística inversa puede ser muy crítica, especialmente en empresas donde el valor de los productos o el ratio de devoluciones son muy elevados. Nadie dudará de que los retos que presenta, suponga una variable estratégica esencial. Si no tienen una visión estratégica sobre la Logística Inversa en el presente, es posible que encuentren problemas en un futuro cercano. Si las empresas dirigieran esfuerzos en esta área, podrían conseguir reducciones significativas en los costos logísticos.

Tabla 4. Actividades del proceso de transformación de residuos de actividades productivas.

\begin{tabular}{ll}
\hline Actividades & Descripción \\
\hline
\end{tabular}

1. Reúso, reventa o redistribución

Donde el producto es de nuevo utilizado sin realizarle procesos o tratamientos adicionales; generalmente sucede en productos que fueron devueltos por los clientes por daños leves en los empaques o productos

Se presenta en diferentes niveles tales como: reparación del producto, restauración de 2. Reprocesamiento un módulo de un producto o remanufactura de una de sus piezas; una vez realizadas estas operaciones los productos reprocesados pueden ser de nuevo utilizados en el mercado con las mismas funcionalidades o en nuevos productos.

De las piezas de los productos materiales de empaque y contenedores reutilizables, los

3. Reciclaje cuales pueden ser reutilizados o aprovechados para la elaboración de otros productos con el fin de ahorrar costos y proteger el medioambiente.

En la cual se destruye el producto y luego se envía a botaderos de basura. Esta última 4. Eliminación actividad suele considerarse como la última opción, debido a que se desecha el producto totalmente, cerrando la posibilidad de usarlo en otros procesos productivos.

\section{DISCUSIÓN}

Los resultados de la investigación documental presentados en la tabla 1 en donde se revisa el concepto de logística inversa y en la tabla 2 se esquematiza las diferencias que existe entre la logística directa y la inversa y en la tabla 3 se analiza la valoración cualitativa de los costos de logística inversa y en la tabla 4 se ilustra el proceso que sigue el proceso de transformación de los residuos industriales.

Por otro lado, a la luz de la revisión documental se delinea los beneficios y los aspectos críticos que existen en el proceso de gestión de logística inversa.

Algunas de las ventajas o beneficios potenciales de la implementación de un programa de Logística Inversa se mencionan a continuación:

Disminución de la "sorpresa" o incertidumbre en la llegada de PFU.

Reaprovechamiento de algunos materiales.

Posibilidad de la empresa de abarcar otros mercados.

Mayor confianza en el cliente al momento de tomar la decisión de compra.

Mejora considerable de la imagen de la empresa ante los consumidores. 
Obtención de información de retroalimentación acerca del producto.

Los aspectos críticos presentes en la gestión de la Logística Inversa se detallan en líneas siguientes:

Se requiere la realización de estudios previos para el establecimiento de políticas de decisión en el tema.

No se trata sólo de una simple manipulación del producto.

Todos los depa8rtamentos de la empresa están relacionados con las actividades que se pretendan implementar de Logística Inversa.

Las entradas a un proceso de Logística Inversa son "impredecibles".

Las inspecciones deben ser realizadas en cada producto de forma individual y minuciosa.

La nueva cadena (inversa) incluye un número de procesos inexistentes en logística directa.

Se debe decidir si la empresa debe realizar las distintas actividades con sus propios recursos o si, por el contrario, requerirá los servicios de un operador especializado.

Las devoluciones en pequeñas cantidades tienden a representar mayores costos al integrarlos al sistema.

\section{REFERENCIAS}

Amit, R. \& Schoemaker, R. (1993). Strategic Assets and Organizational Management. Strategic Management Journal 14(1), 33-46.

Carter C. \& Eltram, L. (2006). Environmental Purchasing; Benchmarking our German Counter Partes. Washington, USA, Willey Library.

Council of Logistics Management (2003). URL www.clml.org

Dowlatshahi, S. (2000). Developing a theory of reverse logistics. Interfaces.

Drucker, P. (1993). La Sociedad Capitalista. Harvard Business Review.

Gaytán, J. (2012) Logística Inversa Una Segunda Oportunidad de Negocio. México DF. ITESM.

Guide, V.D. et al (2000). Supply - chain management for recoverable manufacturing Systems.

Fleischmann, M. (1997). Los modelos cuantitativos para la logística inversa: una revisión. European Journal of Operational Research, 103(1), pp. 1-17.

Krikke, H. (1998). Recovery strategies and reverse logist network desing. Institute for Business Engineering and Technology Application. PhD Thesis. Enschede. The Netherlands University of Twente.

Kroon, Ly Vrijens, G. (1995). Returnable Containers: An Example of Reverse Logistics. International Journal of Physical Distribution \& Logistics Management.

Rogers, D. S., y Tibben-Lembke, R. S. (1999). Dirección hacia atrás: tendencias y prácticas de logística inversa. Going Backwards: Reverse Logistics Trends and Practices. Centro de Manejo de la Logística, Universidad de Nevada, Reno, Estados Unidos.

Ross, D.F. (1998). Competing through supply chain management: creating market - winning routing hazardous wastes. European Journal of Operational Research.

Stock J.R. (2004). Evaluating environmentally conscious business practices. Cousil of Logistics Management. Oak Brook, IL.

Tibben - Lembke, R. (2002). Differences between forward and reverse logistics in a retail environment, supply chain management. An International Journal.

Wadhwa S. y Madaan, J. (2007).Conceptual Framework for Knowledge Management en Reverse Entreprise System. New York, USA. Willey Library. 